\title{
Improving of Crystal Size Distribution Control Based on Neural Network-Based Hybrid Model for Purified Terephthalic Acid Batch Crystallizer
}

\author{
Paisan Kittisupakorn $^{1, a}$, Preeya Somsong ${ }^{1}$, Mohd Azlan Hussain ${ }^{2, b}$ and Wachira Daosud ${ }^{3, c^{*}}$ \\ 1 Department of Chemical Engineering, Faculty of Engineering, Chulalongkorn University, Phayathai Road, \\ Bangkok 10330, Thailand \\ 2 Chemical Engineering Department, University of Malaya, Kuala Lumpur 50603, Malaysia \\ 3 Department of Chemical Engineering, Faculty of Engineering, Burapha University, Longhadbamgsaen \\ Road, Chonburi 20131, Thailand \\ E-mail: apaisan.k@chula.ac.th, bmohd_azlan@um.edu.my, ${ }^{c}$ wachira@buu.ac.th (Corresponding author)
}

\begin{abstract}
A main difficult task in batch crystallization is to control the size distribution of crystal products. Complexity and highly nonlinear dynamic behavior directly affect to model-based control strategies which heavily depend on the rigorous knowledge of crystallization. In this work, neural network-based model predictive control and inverse neural network control strategies are proposed and integrated with an optimization based on neural network-based hybrid model to control temperatures of a purified terephthalic acid batch crystallizer. A neural network-based hybrid model of the batch crystallizer is developed to provide nonlinear dynamic responses used in optimization algorithm for finding an optimal temperature profile related to the quality of a crystal product. Then, the obtained optimal profile is used as set points of the proposed control strategies for improving the crystal product quality. The performances and robustness of the proposed controllers are evaluated in several cases such as for set point tracking and plant/model mismatches. Simulation results show that the neural network-based model predictive control gives the best control performance among the inverse neural network control and a conventional PID controller in all cases.
\end{abstract}

Keywords: Hybrid model, neural network-based model predictive control, crystal size distribution, batch crystallizer.

ENGINEERING JOURNAL Volume 21 Issue 7

Received 27 March 2017

Accepted 7 August 2017

Published 29 December 2017

Online at http://www.engj.org/

DOI:10.4186/ej.2017.21.7.319 


\section{Introduction}

Purified Terephthalic acid (PTA), one of major commodity chemicals, is the principal raw material mainly used for a polyester industry. PTA is produced by the air oxidation of $\mathrm{p}-\mathrm{xylene}$ in acetic acid promoted by cobalt $(\mathrm{Co})$, manganese $(\mathrm{Mn})$, and bromine $(\mathrm{Br})$ catalysts. It is a crystal which contains two carboxylic groups in the paraposition on a benzene ring. The impurities present in PTA crystals are generally intermediates, oxidation by-products, and catalysts [1,2]. Purification of PTA crystals is required to achieve more purity and high-value products.

A crystallization process plays an important role in many industries. In a specialty chemical industry, crystallization is utilized for solid-liquid separation and purification of high-valued chemicals and household products. For the production process of PTA, the crystallization process is used to separate PTA from water and is operated in a batch mode which the quality of final crystal product is usually determined by final time crystal size distribution (CSD) or related properties such as mean crystal size, final seeded crystal size, the final mass of seeded or nucleated crystals, and the variance of the product CSD. Generally, the CSD is directly affected by the non-equilibrium state of the system measured via supersaturation. Since supersaturation is a function of a crystallizer temperature, the slurry temperature is often used as the manipulated variable to control the non-equilibrium driving force of the system [3, 4]. However, to achieve the objective of the control, an optimal condition and effective controllers are required.

Recently, optimization of batch crystallization processes has received considerable attention as it is a useful tool to determine an optimal operating temperature which has a direct effect on the final-time CSD [4, 5]. For example, Miller and Rawling implemented model identification and an open-loop optimal temperature control strategy on a bench-scale potassium nitrate-water system [6]. However, at the moment there was still limited understanding of complex and highly nonlinear systems like the crystallization process, one of the most effective techniques to handle such a situation was an artificial neural network. An obvious advantage of neural network was its universal character in approximating different physical phenomena with similar computational structure. The ability to approximate complex nonlinear relationships from process data without prior knowledge of the model structure makes neural network very attractive to the classical modeling techniques [7]. Daosud et al. improved the purity of acetone using neural network model for model based control algorithm [8]. Kittisupakorn et al. applied neural network model for the prediction of the concentration profile of a hydrochloric acid for hydrochloric acid recovery process and used neural network as a model in control algorithm for a steel pickling process [9].

Model Predictive Control (MPC) is one of model based approaches which can handle most common process characteristics and industrial requirements in a satisfactory way. The key success factor in the use of MPC in these process problems is the existence of accurate process models. However, chemical processes such as this batch crystallization process present the natured nonlinear dynamic behavior and multivariable interactions between variables that cause actually highly difficulty to obtain the accurate model. In this way, neural networks offer alternative nonlinear models for implementing MPC in such as systems [10-13]. The applications of neural networks for chemical process modeling and MPC have also been investigated for SISO systems and iterative multistep neural network predictions in MPC based control for MIMO chemical processes [14-19]. Production of a uniform and reproducible CSD is a main difficulty in batch crystallization $[20,21]$. There are several approaches proposed in the literature; nevertheless, one way to improve the control of CSD is to use supersaturation control (SSC) [22-24], which drives the process within the metastable zone to avoid nucleation. Therefore, model-based approaches, i.e. neural network-based MPC, have been applied for better predictive control [25-27].

In this work, the production of a uniform CSD by supersaturation controlling (SSC) via crystallizer's temperature control which keeps the process within the supersaturated zone is studied. The neural network model for batch crystallization have been developed and their optimal structures have been chosen based on the mean square error (MSE) technique. The obtained optimal neural network structures combined with moment models produced the neural network-based hybrid models and used to obtain the optimal temperature profile by an optimization strategy minimizing the total volume of nucleated fine crystals and maximizing the total volume of the desired crystals growing from seeds. The model predictive control (MPC) based on neural network model and inverse neural network controllers were then applied to control the seeded batch crystallizer of PTA in order to achieve a uniform crystal size distribution. 


\section{Mathematical Models of a Batch Purified Terephthalic Acid Crystallizer}

Batch crystallization models represented by a reduced order moment model of the nuclei and seed classes of crystals to describe the dynamic behavior of the process are given as follows [4]:

$$
\begin{gathered}
\frac{d \mu_{0}^{n}}{d t}=B(t) \\
\frac{d \mu_{i}^{n}}{d t}=i G(t) \mu_{i-1}^{n}(t), \quad i=1,2,3 \\
\mu_{0}^{s}=\text { constant } \\
\frac{d \mu_{i}^{s}}{d t}=i G(t) \mu_{i-1}^{s}(t) \quad i=1,2,3
\end{gathered}
$$

where $\mu_{i}(i=0, \ldots, 3)$, which is the summation of $\mu_{i}^{n}$ and $\mu_{i}^{s}$, respectively, represents the average product quality in terms of the total of number, length, surface area and volume of crystals and $B(t)$ and $G(t)$ are the nucleation and growth rates of crystals, respectively.

To develop the models, following assumptions are made; volume change in the system is negligible, crystal agglomeration or breakage phenomena can be neglected and the crystal density and the super saturation are homogeneous in all parts of the crystallizer, the mass and energy balances describing the changes in the concentration and temperature of solution and cooling temperature in the process can be expressed as Eqs. (5)-(7). The meanings of the letters and symbols are given in the nomenclature.

$$
\begin{gathered}
\frac{d C}{d t}=-3 \rho_{c} k_{v} G(t) \mu_{2}(t) \\
\frac{d T}{d t}=-3 \frac{\Delta H_{c}}{C_{p}} \rho_{c} k_{\nu} G(t) \mu_{2}(t)-\frac{U A}{M C_{p}}\left(T(t)-T_{j}(t)\right) \\
\frac{d T_{j}}{d t}=\frac{F_{j}}{V_{j}}\left(T_{j \cdot p}(t)-T_{j}(t)\right)+\frac{U A}{\rho_{j} V_{j} C_{p j}}\left(T(t)-T_{j}(t)\right)
\end{gathered}
$$

To complete the model of crystallization, the kinetic processes involved determination of rate expressions for crystal nucleation and growth are defined as follows:

$$
\begin{gathered}
B(t)=k_{b} e^{-E_{b} / R T}\left(\frac{C-C_{s}(t)}{C_{c}(t)}\right)^{b} \mu_{3}(t) \\
G(t)=k_{g} e^{-E_{g} / R T}\left(\frac{C-C_{s}(t)}{C_{c}(t)}\right)^{g}
\end{gathered}
$$

where $E_{b}$ is the nucleation activation energy, $E_{g}$ is the growth activation energy, $b$ is an exponent relating nucleation rate to the supersaturation, $g$ is an exponent relating growth rate to the supersaturation, $C_{s}$ is the saturation concentration of the solute, and $\mu_{3}$ is the third moment of the CSD that explains the total volume of crystals.

In this work, the seeded batch crystallizer of PTA is considered [28] and the process parameters are given in Table 1. Equation (10) is used to calculate the saturation concentration corresponding to the solution temperature, $T$ and the definitions of the average crystal size $(L)$ is expressed as Eq. (11).

$$
C_{s}(T)=6 \times 10^{-5} e^{0.0396 T}
$$




$$
L=\frac{\mu_{3}}{\mu_{2}}
$$

Table 1. Model parameters of a seeded batch crystallizer of purified terephthalic acid.

\begin{tabular}{ll}
\hline$b=0.472$ & $A=0.25 \mathrm{~m}^{2}$ \\
$g=0.984$ & $C p=3.86 \mathrm{~kJ} \mathrm{(kg} \mathrm{K})^{-1}$ \\
$k_{b}=6.517 \times 10^{-12} \mathrm{~s}^{-1} \mu \mathrm{m}^{-3}$ & $\left.C p_{j}=4.184 \mathrm{~kJ} \mathrm{(kg} \mathrm{K}\right)^{-1}$ \\
$k_{g}=1.568 \mathrm{~s}^{-1} \mu \mathrm{m}$ & $\varrho_{c}=1.58 \times 10^{-12} \mathrm{~kg} \mathrm{~m}^{-3}$ \\
$E_{b} / \mathrm{R}=-7517 \mathrm{~K}$ & $\varrho_{j}=1000 \mathrm{~kg} \mathrm{~m}^{-3}$ \\
$E_{g} / \mathrm{R}=5.689 \times 10^{-3} \mathrm{~K}$ & $M=27.0 \mathrm{~kg}^{-3}$ \\
$U=1000 \mathrm{~kJ}\left(\mathrm{hr} \mathrm{m}^{2} \mathrm{~K}\right)^{-1}$ & $F_{j}=0.001 \mathrm{~m}^{3} \mathrm{~s}^{-1}$ \\
$\Delta H_{c}=122.758 \mathrm{~kJ} \mathrm{~kg}^{-1}$ & $k_{v}=1.5$ \\
$V_{j}=0.15 \mathrm{~m}^{3}$ & $t_{f}=30 \mathrm{~min}$ \\
\hline
\end{tabular}

\section{Neural Network}

\subsection{Neural network modeling for optimal purpose}

In this work, the purpose of implementing the neural network model (NN1) in the optimization strategy is to predict the dynamic behavior of the batch crystallizer, i.e., the solution concentration and the temperature, when the temperature of the crystallizer changes. The outputs of NN1 are composed of the solution concentration and the crystallizer temperature which are used in the set of moment equation (Eqs.(1)-(4)) as mentioned earlier for finding the total volume of fine and seeded crystals represented by the third moments of crystals $\left(\mu_{3}^{n}\right.$ and $\left.\mu_{3}^{s}\right)$.

The NN1 is designed as a feedforward neural network with the structure consisting of 4 nodes in the input layer and 2nodes at the output layer as shown in Fig. 1. The input layer nodes are the previous and current values of the crystallizer temperature $(T)$ and the solution concentration $(C)$, which directly correspond to the future values of crystallizer temperature and the solution concentration. Before implementing NN1 in the optimal algorithm, it was optimized using the training and validation data sets generated from the theoretical batch crystallizer model (Eqs. (1)-(10)) covering several possible scenarios consisting of the nominal and uncertain cases with plant/model mismatched uncertainty. The changes of overall heat-transfer coefficient, growth activation energy, nucleation activation energy, growth rate coefficient and birth rate coefficient are represented as plant/model mismatches. In addition, the manipulated variable (the set point of the jacket temperature) is adjusted as step changes and random changes in ranges of $270-400 \mathrm{~K}$. The operational time is kept constant at 30 minutes and the sampling time of data is 0.1 minutes.In the normalization step, all data are scaled in the range of the minimum and maximum value. Details of the procedure for obtaining the feedforward neural network models are explained in research [9].

The optimal NN structure is obtained by finding the number of nodes in the hidden layer where the NN1 has been developed based on the Lenvenberg-Marquardt training algorithm [9]. The sigmoid function is used as the activation functions of the nodes in the hidden layer and the linear transfer function used for its output layer. The objective of the neural network training is to minimize an error between predicted neural network values and actual targeted values ( $T$ and $C$ ), which is defined by the mean square error (MSE). From simulation results, the optimal architecture of the NN1 for the prediction of the solution concentration and the temperature of crystallizer consists of one hidden layer with 4 nodes. The details of NN1 is shown in Table 2. The selected NN model, NN1, is used in the dynamic optimization algorithm for prediction of the crystallizer temperature and the solution concentration of future values described in the next section, for the dynamic optimization. 
Table 2. The details of neural network models.

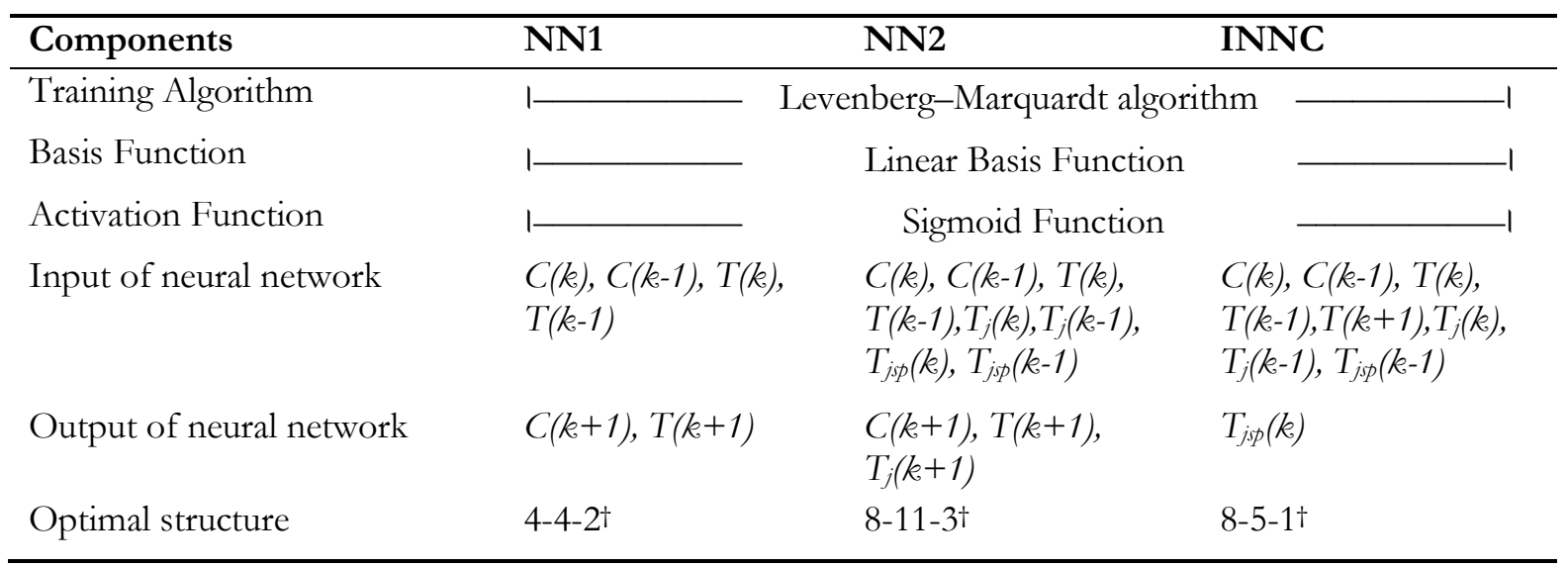

†(Input nodes-Hidden nodes-Output nodes)

\subsection{Dynamic Optimization}

For the industrial crystallization, one of main problems that cause difficulties in downstream operation such as filtration and drying is the fine crystals. Thereby the objective of this dynamic optimization is to determine the optimal crystallizer temperature profile minimizing the total volume of nucleated fine crystals $\left(\mu_{3}^{n}\right)$ and maximizing the total volume of the desired crystals growing from seeds $\left(\mu_{3}^{s}\right)$ at the end of the batch operation which represents the efficiency of the batch crystallizer. In this work, three optimal problems: OPT1, OPT2 and OPT3 corresponding to the 3 different objectives of dynamic optimization are proposed to provide the optimal crystallizer temperature profile in the purified terephthalic acid batch crystallizer. These optimal problems are applied in the optimization algorithm and the predicted process behaviors provided by neural network-based hybrid model. The structure of optimization algorithm is shown in Fig. 1, which uses sequential quadratic programming to solve the optimization problems with respect to $T$. The detail of each optimal problem is described as follows.

- Optimal problem: OPT1

This optimal problem describes maximizing the total volume of the desired crystals growing from seeds $\left(\mu_{3}^{s}\right)$ at the end of batch operation, expressed as follows:

$$
\underset{T(k+1)}{\operatorname{Maximize}} \mu_{3}^{s}\left(t_{f}\right)
$$

- Optimal problem: OPT2

The OPT2 provides the problem of minimizing total volume of nucleated fine crystals $\left(\mu_{3}^{n}\right)$ at the end of batch operation, which is defined as follows:

$$
\underset{T(k+1)}{\operatorname{Minimize}} \mu_{3}^{n}\left(t_{f}\right)
$$

- Optimal problem: OPT3

The last optimal problem, OPT3, attempts to minimize the total volume of nucleated fine crystals $\left(\mu_{3}^{n}\right)$ and at the same time maximize the total volume of the desired crystals growing from seeds $\left(\mu_{3}^{s}\right)$ at the end of batch operation. Therefore, the optimal problem takes the form

$$
\underset{T(k+1)}{\operatorname{Minimize}} \mu_{3}^{n}\left(t_{f}\right)-\mu_{3}^{s}\left(t_{f}\right)
$$


All of the optimal problems are subject to the following constraints,

NN1 and the moment model of batch crystallizer (Eq. (1)-(4))

$$
\begin{aligned}
& C_{s} \leq C(\mathrm{k}) \\
& 400 \mathrm{~K} \leq T(\mathrm{k}) \leq 523 \mathrm{~K} \\
& 170 \mu \mathrm{m} \leq L_{w} \leq 190 \mu \mathrm{m}
\end{aligned}
$$

where the lower and upper bounds on the solution temperature are chosen as 400 and $523 \mathrm{~K}$, the lower and upper bounds on the average crystal size are 170 and $190 \mu \mathrm{m}$ respectively corresponding to the desirable quality of the crystal product and the final batch time $\left(t_{f}\right)$ is 30 minutes.

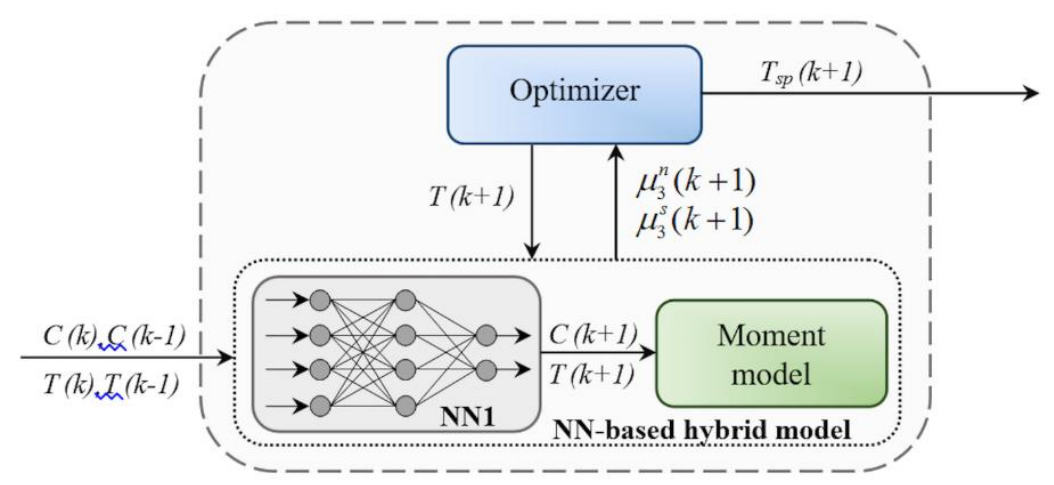

Fig. 1. Optimization structure based on NN-based hybrid model.

After the optimization process, the optimal crystallizer temperature profile is obtained and used as the set point of the designed controllers (neural network model based predictive control, inverse neural network control and PID). The simulation results of optimization with proposed optimal problems (OPT1, OPT2, and OPT3) show that the optimization using OPT3 gives the best performance with maximum average crystal size and volume of the desired crystals growing from seeds $\left(\mu_{3}^{s}\right)$ as seen in Table 3 while Fig. 2 shows the CSD of OPT3 optimization. Figure 2 shows a division of the CSD into two groups which are the nucleated crystals and the crystals growing from seeds. The total volume of nucleated crystals $\left(\mu_{3}^{n}\right)$, the volume of seeded crystals $\left(\mu_{3}^{s}\right)$ and the average crystal size $(L)$, which define the quality of product are given in Table 3.

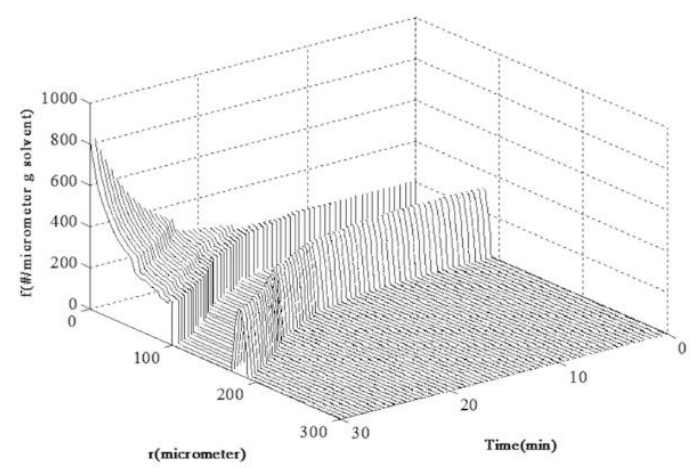

Fig. 2. The crystal size distribution of OPT3 optimization. 
Table 3. The result of dynamic optimization with proposed optimal problems.

\begin{tabular}{|c|c|c|c|c|c|}
\hline \multirow{2}{*}{$\begin{array}{l}\text { Optimal } \\
\text { problem }\end{array}$} & \multicolumn{5}{|c|}{ Properties of product } \\
\hline & $L^{n}(\mu \mathrm{m})$ & $L^{s}(\mu \mathrm{m})$ & $L(\mu \mathrm{m})$ & $\square^{n}\left(\mu m^{3}\right.$ g solvent $\left.^{-1}\right)$ & $\square^{s}\left(\mu \mathrm{m}^{3} \mathrm{~g}\right.$ solvent $\left.{ }^{-1}\right)$ \\
\hline OPT 1 & 37.705 & 179.786 & 174.608 & $2.165 \times 10^{8}$ & $6.240 \times 10^{9}$ \\
\hline OPT 2 & 34.864 & 177.381 & 173.409 & $1.833 \times 10^{8}$ & $5.692 \times 10^{9}$ \\
\hline OPT 3 & 62.575 & 180.972 & 176.245 & $2.246 \times 10^{9}$ & $5.531 \times 10^{10}$ \\
\hline
\end{tabular}

\section{Neural Network Controller Design}

In this section, the controllers are designed based on two strategies which are the NN-based model predictive control (NNMPC) and inverse neural network control (INNC) to control the solution temperature of the batch crystallizer corresponding to the desirable quality of the crystal product: to minimize the total volume of nucleated fine crystals and maximize the total volume of the desired crystals growing from seeds at the end of batch operation. The details of each controller are described as follows.

\subsection{NN-based Model Predictive Control (NNMPC)}

For this control strategy, NN feed forward model is applied as a predictor to predict the future values of the process behavior over a prediction horizon ( $p$ ) within a MPC algorithm. The NN in this algorithm, NN2, is obtained by the procedure for developing neural network models as defined in research [9]. The training and validation data sets are obtained by selecting appropriate signals from the simulation of the purified terephthalic acid batch crystallizer by solving Eqs.(1)-(10) covering several possible scenarios. These equations are solved to obtain the process states according to various step changes in the manipulated variables, jacket temperature set points $\left(T_{j p p}\right)$. The inputs to the NN2 selected are the previous and current value of the input variables $\left(C, T, T_{j}\right.$ and $\left.T_{j \cdot p}\right)$ which highly effect the output state variables of the process $(C$, $T$ and $\left.T_{j}\right)$.The NN2 model can be expressed as the function of inputs as shown below:

$$
C(k+1), T(k+1), T_{j}(k+1)=f\left(C(k), C(k-1), T(k), T(k-1), T_{j}(k), T_{j}(k-1), T_{j j p}(k), T_{j j p}(k-1)\right)
$$

The applicable structure is selected by the minimum MSE method [9]. The hidden nodes of hidden layer are varied in various quantities. The MSE error is then determined and the nodes that give minimum MSE value is selected for determining the final number of hidden nodes. Therefore, the optimal structure of the NN2 is 8-11-3 configuration. The component of the NN2 is shown in Table 2.

After NN2 predicts future outputs several steps over the prediction horizon $(p)$, the predicted outputs are passed to the optimization routine which produces the present and future control signals $\left(T_{j p p}(k+1), \ldots, T_{j p p}(k+m)\right)$. They are selected by minimizing the sum of squares of the errors between the predicted outputs and the setpoint values (the temperature profile getting from OPT3 optimization) evaluated over the prediction horizon (objective function, Eq. (16)). The objective function of the NNMPC strategy can be written in the form as follows:

$$
\underset{T_{j p \phi}(k+1), \ldots, T_{j p p}(k+m)}{\operatorname{Minimize}} \sum_{i=1}^{p}\left\{W_{1}\left[T_{s p}(k+i)-T(k+i)\right]^{2}+W_{2}\left[\Delta T_{j p \phi}\right]^{2}\right\}
$$

subject to

the optimal feed forward neural network model , NN2

$$
\begin{aligned}
& 270 \mathrm{~K} \leq T_{j p p}(k+i) \leq 330 \mathrm{~K}, i=1,2,3, \ldots, p \\
& T(k+p)=T_{s p}(k+p)
\end{aligned}
$$

where $p$ is a parameter specifying the prediction horizon; $T_{s p}$ is the required set point (the optimal set point obtained by OPT 3 optimization), $T$ is the solution temperature and $W_{1}, W_{2}$ are weighting parameters. The values for the parameters, $p ; m$ (control horizon) and $W_{1}, W_{2}$ for this strategy are provided by various trials through simulations. Then, $p$ is set at five and $W_{1}, W_{2}$ are chosen as the identity vector. The control horizon 
$(m)$ is set as three. Sequential quadratic programming is used to solve the optimization problem by minimizing the objective function in Eq. (16) with respect to $T_{j p p}$ and to produce a solution constrained within the desired ranges. The algorithm of NNMPC is shown in Fig. 3.

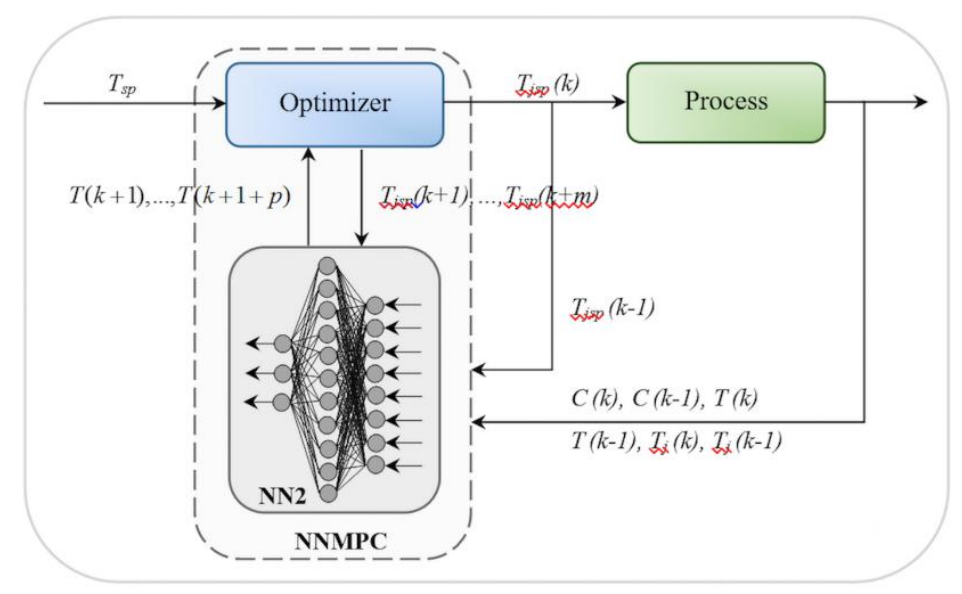

Fig. 3. NNMPC strategy.

As shown in Fig. 3, NN2 is used to predict the next $p$ values of the process outputs $(T(k+1), \ldots, T(k+1+p))$ by iterative prediction. After that the set of manipulated variables $\left(T_{j \cdot p}(k+1), \ldots, T_{j \cdot p}(k+m)\right)$ are calculated using the process outputs predicted in previous step by the sequential quadratic programming procedure (SQP). SQP procedure are iterative at each sample time. Although the solution of Eq. (16) yields a vector of future control moves $\left(T_{j p p}(k+m)\right)$ at each sample interval, only the first one $\left(T_{j p p}(k)\right)$ is implemented to adjust the solution temperature to the desired set points.

\subsection{Inverse Neural Network Control (INNC)}

Math The feed forward inverse neural network, INNC, is designed and used for the control the solution temperature in neural network inverse control strategy. This strategy consists of the neural network inverse model that acts as the controller placed in series with the process under control as shown in Fig. 4 INNC is fed with the required future $(T(k+1))$ or reference output together with the past inputs and the past output variables to predict the current input or control action $\left(T_{j p p}(k)\right)$. Then, the assignment of the input nodes consists of the previous and current values of the input variables $\left(C, T, T_{j}\right.$ and $\left.T_{j p p}\right)$ the desired value of the process output, $T(k+1)$, which corresponds to the required set point or reference signal. The output node of the neural network model consists of the manipulated variable for the batch crystallizer, $T_{j p p}(k)$. Training and validation data sets are obtained by selecting signals from the simulation of the process models by solving Eqs. (1)-(10) which are solved to obtain the process states according to various changes in the manipulated variable $\left(T_{j p p}\right)$. Mathematically, the inverse models are expressed as the function of inputs to the model as shown below:

$$
T_{j p \phi}(k)=f\left(C(k), C(k-1), T(k), T(k-1), T(k+1), T_{j}(k), T_{j}(k-1), T_{j p \phi}(k-1)\right)
$$

The defined neural networks are trained with the Levenberg-Marquardt method where the common objective is to reduce the error between the neural network predicted value and the actual targeted value. The detail of procedure for obtaining the inverse neural network models are define in research [29].The optimum structures is selected by the minimum MSE method [29]. Based on the minimizing MSE error values, it is found that 5 hidden nodes appear to be the best to be applied for the inverse models which will be used as controller in the control strategy. The component of the INNC is indicated in Table 2.

In the control strategy, the neural network inverse models trained as described above are utilized to predict the manipulated jacket temperature set point to bring the process to desired conditions, as shown in Fig. 4. Normally, the prediction of the controller action, i.e. manipulated variable effectively make the value 
of the controlled variable, $T(k+1)$, track the set point accordingly. The control performance is tested under the nominal case and with model mismatch added into the process. The simulation results and discussion of this control studies are described in the next section.

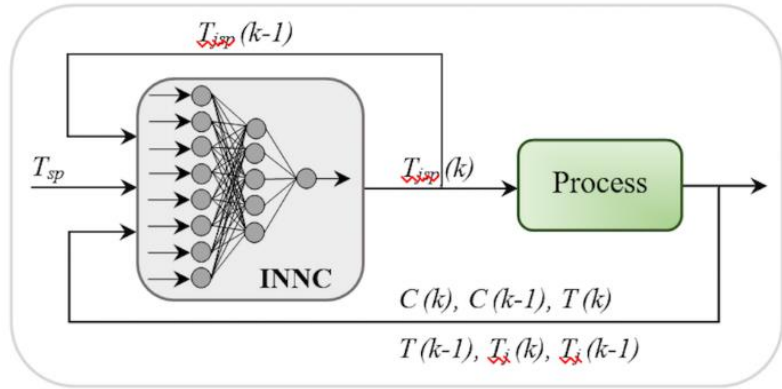

Fig. 4. Inverse neural network control strategy.

\section{Results and Discussion}

The proposed NNMPC and INNC strategies are applied to control the solution temperature in the purified terephthalic acid batch crystallizer at an optimal temperature set point (obtained by NN-based hybrid model optimization) by determining the set point of a manipulated variable $\left(T_{j p p}\right)$. Simulations are divided into two cases of control studies, which are nominal and plant/model mismatch cases.

For the nominal case, the controllers are designed to bring the solution temperature in the batch crystallizer to the desired value. The desired set points are the optimal step change temperature profile at $400 \mathrm{~K}$ to $523 \mathrm{~K}$, which are calculated by the NN-based hybrid model optimization corresponding to minimize $\mu_{3}^{n}$ and at the same time maximizes $\mu_{3}^{s}$ at the end of batch operation. The control results (Fig. 5(a)) show that, NNMPC drives the solution temperature to track the set points without overshoot and oscillations. The satisfactory performance obtained is due to the accurate representation of the nonlinear dynamics of the process by the neural network model. The results (Fig. 5(b)) indicate that INNC can bring the temperature to the set points and give minimal overshoot without offsets. However, drastic change of the manipulated variable and oscillation at the initial state when changing of set points are remarkably observed. The PID controller tuned using the Ziegler-Nichols closed loop method with subsequent fine tuning gives poor performances as displayed in Fig. 5(c) because of the nonlinear dynamics exhibited by the batch crystallizer leading to overshoot and oscillation of the controlled variables and drastic adjustment of the manipulated variables. Control performances of the controllers are evaluated using the integral absolute error (IAE) as summarized in Table 4. It was found that the NNMPC strategy gives the best results among INNC and PID controllers in term of the least IAE values since there are no overshoot, oscillation and offset.

For the plant/model mismatch cases, overall heat transfer coefficient $(U)$; nucleation activation energy $\left(E_{b}\right)$; growth activation energy $\left(E_{g}\right)$; birth rate coefficient $\left(k_{b}\right)$ and growth rate coefficient $\left(k_{g}\right)$ are subject to uncertainties from their nominal values by $30 \%$. Fig. 6 shows the results of the NNMPC, INNC and PID controllers with subjecting to $30 \%$ decrease in the overall heat transfer coefficient. The figures illustrate that the NNMPC strategy can bring the temperatures to the set points by gradually decreasing the jacket temperatures which gives smooth control response. The INNC strategy can control the temperatures close to their required values by drastically decreasing the jacket temperatures $\left(T_{j}\right)$, while the PID control strategy cannot bring the temperatures to the set points at each step of temperature set points because of rigorous changing of the manipulated variables. Table 4 shows the IAE values of NNMPC, INNC and PID in this case. They indicate that NNMPC is much more robust and gives the best control performance among INNC and PID controllers in all cases. The robustness of the NNMPC can be explained by the fact that the obtained NN model (NN2) for the use in the NNMPC strategy trained with the wide range of operating conditions together with the optimization for obtaining the manipulated profiles in MPC algorithm. On the other hand, the PID controller gives the worst robustness as it cannot handle with these mismathces. 

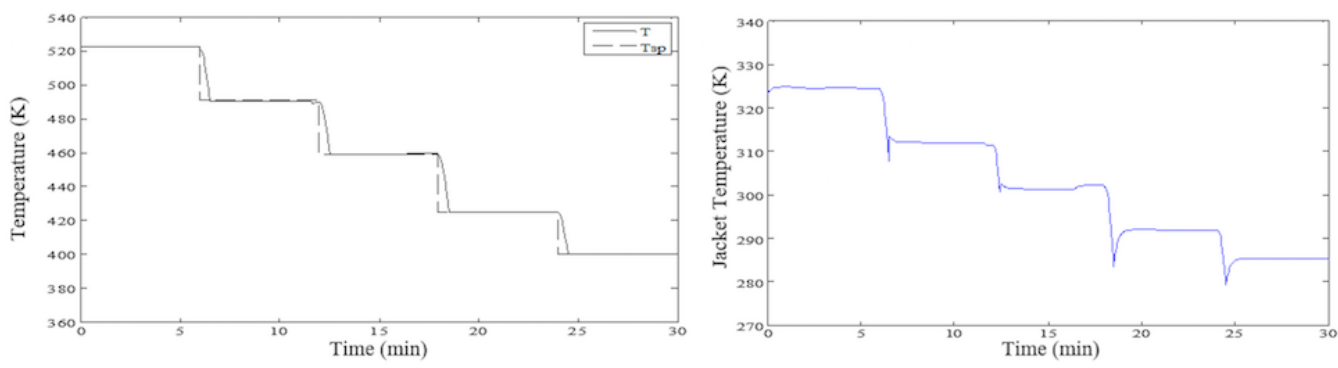

(a)
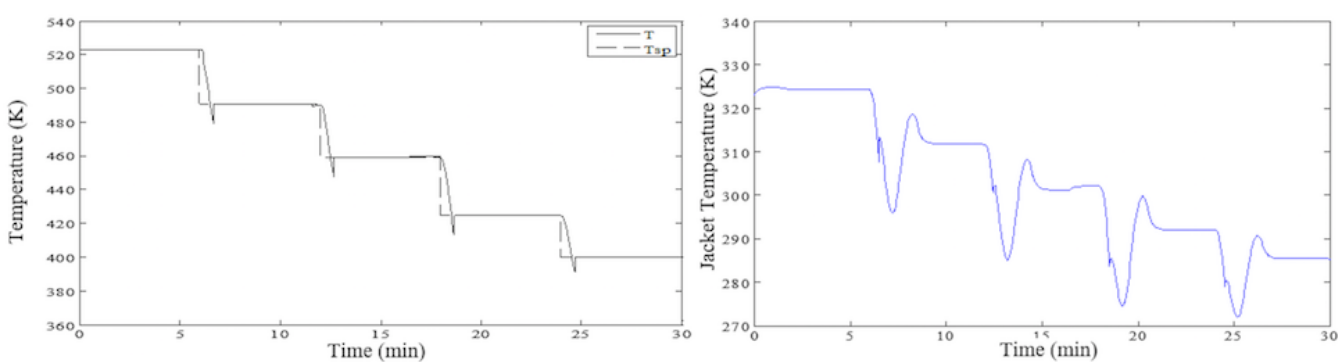

(b)
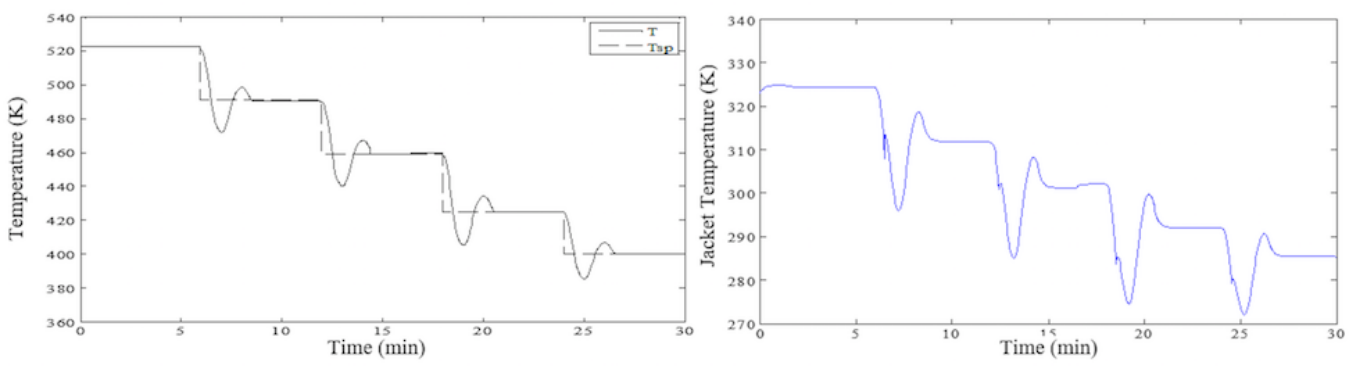

(c)

Fig. 5 Temperature control in the batch crystallizer under a nominal case: (a) NNMPC, (b) INNC and (c) PID.

Table 4. Performance comparisons of NNMPC, INNC and PID.

\begin{tabular}{llll}
\hline Condition & \multicolumn{3}{c}{ IAE } \\
\cline { 2 - 4 } & NNMPC & INNC & PID \\
\hline Nominal case & 164.74 & 168.81 & 169.42 \\
Model mismatch case $(30 \%)$ & & & \\
Decreasing of $U$ & 173.62 & 175.40 & 182.36 \\
Increasing of $k_{b}$ & 167.18 & 170.02 & 171.12 \\
Decreasing of $k_{g}$ & 170.05 & 170.71 & 171.52 \\
Increasing of $E_{b}$ & 175.30 & 177.93 & 179.12 \\
Decreasing of $E_{g}$ & 172.60 & 180.34 & 184.09 \\
Increasing of $E_{b}, k_{g}$ with decreasing of $U, E_{g}, k_{b}$ & 174.54 & 178.81 & 177.61 \\
\hline
\end{tabular}



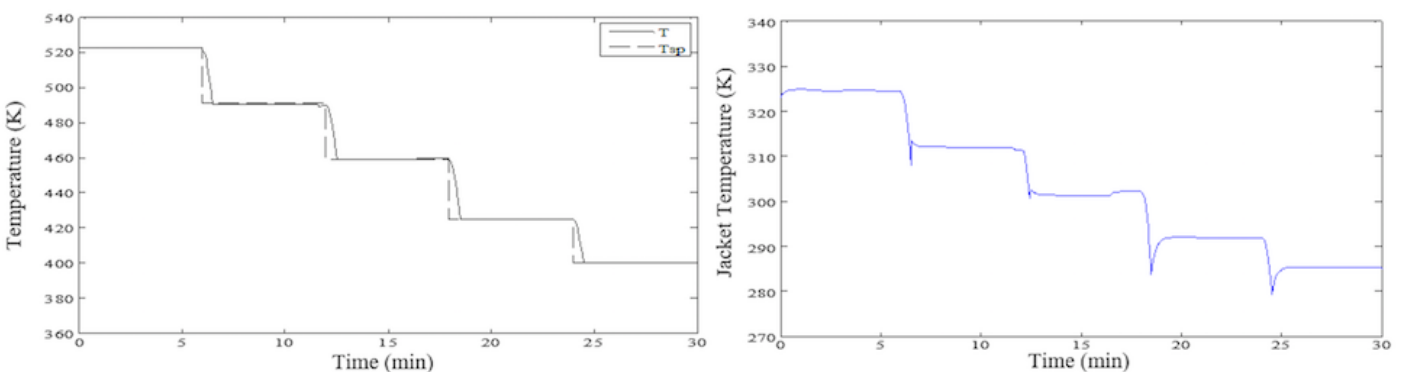

(a)
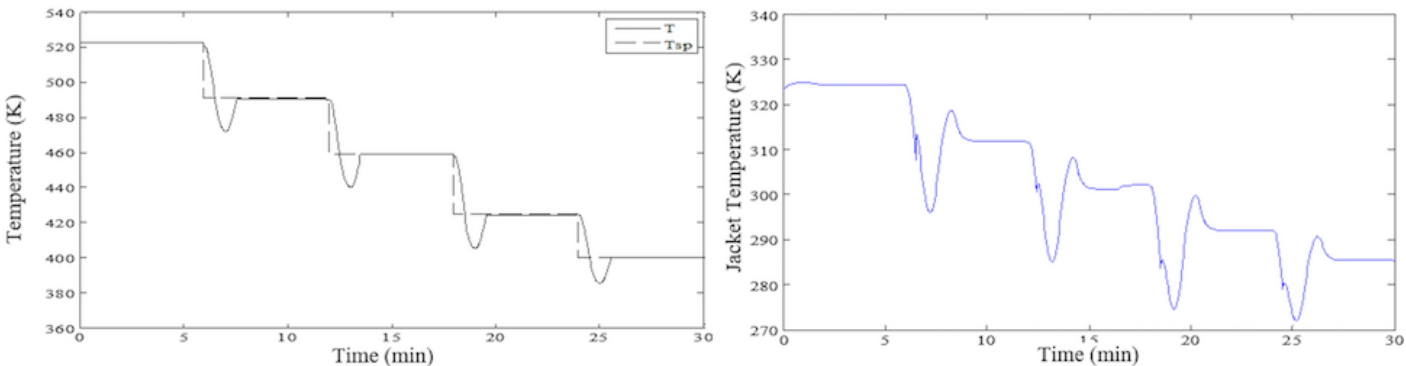

(b)
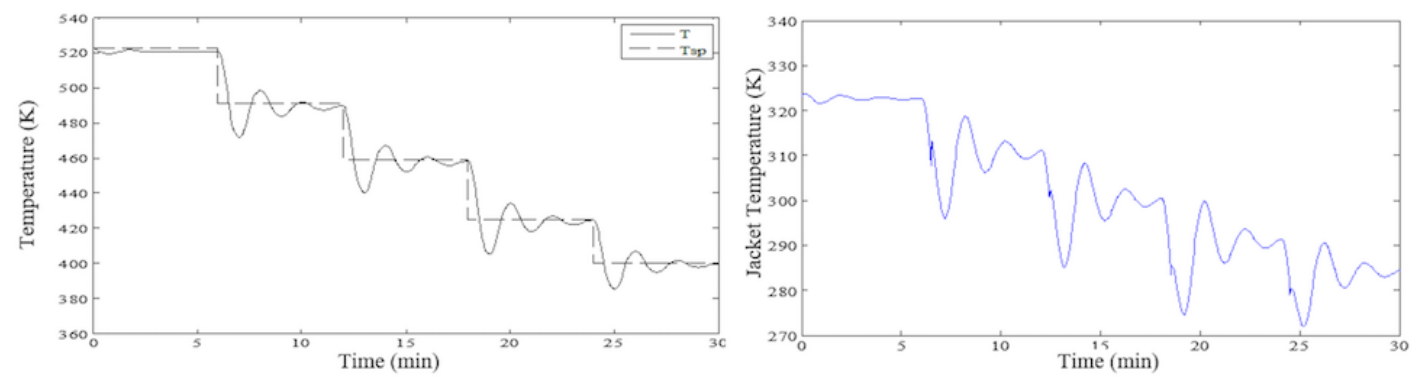

(c)

Fig. 6. Temperature control in the batch crystallizer under the model mismatch case $(30 \%$ decrease of the overall heat transfer coefficient, $U$ ): (a) NNMPC, (b) INNC and (c) PID control.

\section{Conclusions}

Model-based control strategies, NNMPC and INNC, have been proposed and applied with the optimization based on NN-based hybrid model to control the temperatures in the purified terephthalic acid batch crystallizer. The hybrid model based optimization has been implemented to find an optimal temperature profile corresponding to minimize a total volume of nucleated fine crystals $\left(\mu_{3}^{n}\right)$ and at the same time to maximize a total volume of the desired crystals growing from seeds $\left(\mu_{3}^{s}\right)$ at the end of batch operation. The neural network models of the batch crystallizer have been developed for two different purposes: to be a NN model used in the optimization algorithm to predict the future process response and to be a NNMPC control strategy. It was observed that NNMPC can bring the control variables to their set points without oscillations in all cases, i.e., set point tracking case and model mismatch cases. Comparison of control performances among NNMPC, INNC and conventional PID controllers indicated that NNMPC was more robust than the others and gave the best control results in all cases. These results validated the robustness of the NNMPC control strategy which could be applicable for enhancement of crystal size distribution control of the purified terephthalic acid batch crystallizer. 


\section{Acknowledgement}

Support from the Faculty of Engineering, Burapha University under the contract \#23/2555, The Institutional

Research Grant (The Thailand Research Fund), IRG 5780014 and Chulalongkorn University (Contract No. RES_57_411_21_076) are gratefully acknowledged.

\section{References}

[1] P. M. Brown, M. Marquering, and A. S. Myerson, "Purification of terephthalic acid by crystal aging," Ind. Eng. Chem., vol. 29, pp. 2089-2093, 1990.

[2] Q. Wang, Y. Cheng, L. Wang, H. Xu, and X. Li, "Isothermal seeded semicontinuous reactive crystallization of crude terephthalic acid crystals," Ind. Eng. Chem., vol. 47, pp. 5861-5870, 2008.

[3] Q. Hu, S. Rohani, D. X. Wang, and A. Jutan, "Optimal control of a batch cooling seeded crystallizer," Powder. Technol., vol. 156, pp. 170-176, 2005.

[4] W. Paengjuntuek, A. Arpornwichanop, and P. Kittisupakorn, "Product quality improvement of batch crystallizers by a batch-to-batch optimization and nonlinear control approach," Chem. Eng. J., vol. 139, pp. 344-350, 2008.

[5] W. Paengjuntuek, A. Arpornwichanop, and P. Kittisupakorn, "Optimization and nonlinear control of a batch crystallization process," J. Chin. Inst. Chem. Eng., vol. 39, pp. 249-256, 2008.

[6] S. M. Miller and J. B. Rawling, "Model identification and control strategies for batch crystallizer," AlChE. J., vol. 40, pp. 1312-1327, 1994.

[7] J. M. Ali, M. A. Hussain, M. O. Tade, and J. Zhang, "Artificial Intelligence techniques applied as estimator in chemical process systems-A literature survey," Expert. Syst. Appl., vol. 42, pp. 5915-593, 2015.

[8] W. Daosud, K. Jariyaboon, P. Kittisupakorn, and M. A. Hussain, "Neural network based model predictive control of batch extractive distillation process for improving purity of acetone," Engineering Journal, vol. 20, no. 1, pp. 47-59, 2016.

[9] P. Kittisupakorn, P. Thitiyasook, M. A. Hussain, and W. Daosud, "Neural network model predictive control for a steel pickling process," J. Process. Control., vol. 19, pp. 579-590, 2009.

[10] G. Lightbody, P. O’Reilly, G. W. Irwin, K. Kelly, and J. McCormick, "Neural modelling of chemical plant using MLP and b-spline networks," Control. Eng. Pract., vol. 5, no. 11, pp. 1501-1515, 1997.

[11] S. K. Doherty, J. B. Gomm, and D. Williams, "Experiment design considerations for non-linear system identification using neural networks," Comput.ChemEng., vol. 21, no. 3, pp. 327-346, 1997.

[12] D. M. Himmelblau, "Application of artificial neural networks in chemical engineering," Korean. J., Chem. Eng., vol. 17, no. 4, pp. 373-392, 2000.

[13] P. Kittisupakorn, N. Polruksa, and W. Daosud, "Neural networks model for the estimation of melt flow rate and density of polymers," in Proceedings of the Ninth APCChE Congress and CHEMECA, Christchurch, New Zealand, 2002.

[14] S. Li and Y. Li, "Model predictive control of an intensified continuous reactor using a neural network Wiener model," Neurocomputing., vol. 185, pp. 93-104, 2016.

[15] N. Sharma and K. Singh, "Model predictive control and neural network predictive control of TAME reactive distillation column," Chem. Eng. Process., vol. 56, pp. 9-21, 2012.

[16] M. A. Hosen, M. A. Hussain, and F. S. Mjalli, "Control of polystyrene batch reactors using neural network based model predictive control (NNMPC): An experimental investigation," Control. Eng. Pract., vol. 19, pp. 454-467, 2011.

[17] B. C. Ng, I. Z. M. Darus, H. Jamaluddin, and H. M. Kamar, "Application of adaptive neural predictive control for an automotive air conditioning system," Appl. Therm. Eng., vol. 73, pp. 1244-1254, 2014.

[18] P. Kittisupakorn, T. Charoenniyom, and W. Daosud, "Hybrid neural network controller design for a batch reactor to produce methyl methacrylate," Engineering Journal, vol. 18, no.1, pp. 145-162, 2014.

[19] W. Daosud, J. Thampasato, and P. Kittisupakorn, "Neural network based modeling and control for a batch heating/cooling evaporative crystallization process," Engineering Journal, vol. 21, no. 1, pp. $127-$ $144,2017$.

[20] R. D. Braatz, "Advanced control of crystallisation processes," Ann.Rev.Control., vol. 26, pp. 87-99, 2002.

[21] C. Wibowo and K. M.Ng, "Operational issues in solids processing plants: systems view," AIChE. J., vol. 47, pp. 107-125, 2001. 
[22] N. Doki, H. Seki, K. Takano, H. Asatani, M. Yokota, and N. Kubota, "Process control of seeded batch cooling crystallisation of the metastable alpha-form glycine using an in-situ ATR-FTIR spectrometer and an in-situ FBRM particle counter," Cryst. Growth. Des., vol. 4, no, 949-953, 2004.

[23] M. Fujiwara, Z. K. Nagy, J. W. Chew, and R. D. Braatz, "First-principles and direct design approaches for the control of pharmaceutical crystallization," J. Process. Control., vol. 15, pp. 493-504, 2005.

[24] Z. Q. Yu, P. S. Chow, and B. H. T. Reginald, "Seeding and constant-supersaturation control by ATRFTIR in anti-solvent crystallization," Org. Process. Res. Dev., vol. 10, no.717-722, 2006.

[25] P. A. Larsen, D. B. Patience, and J. B. Rawlings, "Industrial crystallisation process control," IEEE.Control.Syst. Mag., vol. 26, pp. 70-80, 2006.

[26] J. D. Ward, D. A. Mellichamp, and M. F. Doherty, "Choosing an operating policy for seeded batch crystallization,” AIChE. J., vol. 52, pp. 2046-2054, 2006.

[27] M. Grosso, O. Galan, R. Barratti, and J. A. Romagnoli, "A novel approach for the prediction of PSD in anti-solvent mediated crystallization," Chem. Eng. Sci., vol. 27, pp. 291-296, 2009.

[28] Q. Wang, Y. Cheng, L. Wang, H. Xu, and X. Li, "Isothermal seeded semicontinuous reactive crystallization of crude terephthalic acid crystal," Ind. Eng. Chem., vol. 47, pp. 5861-5870, 2008.

[29] W. Daosud, P. Thitiyasook, A. Arpornwichanop, P. Kittisupakorn, and M. A. Hussain, "Neural network inverse model-based controller for the control of a steel pickling process," Comput.Chem.Eng., vol. 29, pp. 2110-2119, 2005. 